\title{
Tenacibaculum litopenaei sp. nov., isolated from a shrimp mariculture pond
}

Correspondence

Wen-Ming Chen

p62365@ms28.hinet.net
Shih-Yi Sheu, ${ }^{1}$ Kuan-Yin Lin, ${ }^{2}$ Jui-Hsing Chou, ${ }^{3}$ Poh-Shing Chang, ${ }^{4}$ A. B. Arun, ${ }^{3}$ Chiu-Chung Young ${ }^{3}$ and Wen-Ming Chen ${ }^{2}$

\author{
${ }^{1}$ Department of Marine Biotechnology, National Kaohsiung Marine University, Kaohsiung, \\ Taiwan \\ ${ }^{2}$ Laboratory of Microbiology, Department of Seafood Science, National Kaohsiung Marine \\ University, No. 142, Hai-Chuan Rd, Nan-Tzu, Kaohsiung City 811, Taiwan \\ ${ }^{3}$ Department of Soil Environmental Science, College of Agriculture and Natural Resources, \\ National Chung Hsing University, Taichung, Taiwan \\ ${ }^{4}$ Department of Aquaculture, National Kaohsiung Marine University, Kaohsiung, Taiwan
}

The genus Tenacibaculum Suzuki et al. 2001 is a member of the family Flavobacteriaceae (Reichenbach, 1992a, b; Bernardet et al., 1996, 2002). It currently contains eight species isolated from different marine sources. Tenacibaculum maritimum and T. ovolyticum [previously Flexibacter maritimus (Wakabayashi et al., 1986) and Flexibacter ovolyticus (Hansen et al., 1992)] were isolated from diseased red sea bream fingerling and halibut egg, respectively. Tenacibaculum mesophilum and T. amylolyticum were isolated from sponge homogenate and the surface of marine macroalgae, respectively (Suzuki et al., 2001). Tenacibaculum skagerrakense was isolated from seawater (Frette et al., 2004), while Tenacibaculum lutimaris, T. litoreum and T. aestuarii were isolated from tidal flat sediment (Yoon et al., 2005; Choi et al., 2006; Jung et al., 2006).

In August 2000, in the course of a study aiming at isolating chitinolytic bacteria, a surface water sample was collected from a shrimp (Litopenaeus vannamei) mariculture pond located in Pingtung County, southern Taiwan. The water sample was serially diluted and plated on chitin salt agar plates and incubated at $28^{\circ} \mathrm{C}$. Chitin salt medium contained

The GenBank/EMBL/DDBJ accession number for the 16S rRNA gene sequence of strain $B-I^{\top}$ is DQ822567. $\left(\mathrm{l}^{-1}\right) 5 \mathrm{~g}$ colloidal chitin, $30 \mathrm{~g} \mathrm{NaCl}, 1.0 \mathrm{~g} \mathrm{MgSO}_{4} .7 \mathrm{H}_{2} \mathrm{O}$, $0.2 \mathrm{~g} \mathrm{~K}_{2} \mathrm{HPO}_{4}, 0.5 \mathrm{~g} \mathrm{CaCl}_{2} .2 \mathrm{H}_{2} \mathrm{O}$ and $1.0 \mathrm{ml}$ trace element solution. The $\mathrm{pH}$ was adjusted to 7.5. Colloidal chitin was prepared from commercial chitin (Ohka Chemical) as described by Chang et al. (2004). The trace element solution contained $\left(\mathrm{l}^{-1}\right) 2.8 \mathrm{~g} \mathrm{H}_{3} \mathrm{BO}_{3}, 2.0 \mathrm{~g} \mathrm{MnSO}_{4} .4 \mathrm{H}_{2} \mathrm{O}, 0.2 \mathrm{~g}$ $\mathrm{ZnSO}_{4} .7 \mathrm{H}_{2} \mathrm{O}, 0.08 \mathrm{~g} \mathrm{CuSO}_{4} .5 \mathrm{H}_{2} \mathrm{O}, 0.1 \mathrm{~g} \mathrm{FeCl}_{3}$ and $0.14 \mathrm{~g}$ $\mathrm{Na}_{2} \mathrm{MoO}_{4} \cdot 2 \mathrm{H}_{2} \mathrm{O}$ (Chang et al., 2004). Agar plates were prepared by adding $1.5 \%(\mathrm{w} / \mathrm{v})$ Bacto agar (BD Difco) to the chitin salt medium. A bacterial strain that showed large clear zones around the colonies resulting from chitin degradation was isolated and designated $\mathrm{B}-\mathrm{I}^{\mathrm{T}}$. Strain $\mathrm{B}-\mathrm{I}^{\mathrm{T}}$ was further subcultivated on marine agar 2216 (MA; BD Difco) and the organism was subjected to a polyphasic taxonomic study.

The morphology of bacterial cells was observed during the lag, exponential and stationary phases of growth under a phase-contrast microscope. Gliding motility was tested as described by Bowman (2000) and Bernardet et al. (2002). Flagellar staining was performed using the Spot Test flagella stain (BD Difco). The Gram reaction was assessed using the Gram stain set (BD Difco) and the Ryu non-staining $\mathrm{KOH}$ method (Powers, 1995). Accumulation of poly- $\beta$-hydroxybutyrate granules was observed by light microscopy after staining cells with Sudan black. Cellular pigments were 
extracted from cultures grown on MA by using an acetone/ methanol mixture $(7: 2, \mathrm{v} / \mathrm{v})$ and absorption spectra were determined with a scanning UV/visible spectrophotometer. The presence of flexirubin-type pigments was investigated as described by Reichenbach (1992a) and Bernardet et al. (2002). The $\mathrm{pH}$ range for optimum growth was examined in marine broth $2216(\mathrm{MB})$ using appropriate biological buffers (pH 3-11) (Chung et al., 1995). The $\mathrm{pH}$ was adjusted prior to sterilization; post-sterilization controls revealed that only minor changes in $\mathrm{pH}$ had occurred. Requirement for $\mathrm{NaCl}$ was determined using nutrient broth containing no $\mathrm{NaCl}$ and $0.5 \%$ and $1.0-10.0 \% \mathrm{NaCl}(\mathrm{w} / \mathrm{v}$, at $1.0 \%$ intervals $)$. The temperature range for growth $(4,10,15,20,25,30,35$, 39 and $40{ }^{\circ} \mathrm{C}$ ) was examined in $\mathrm{MB}$ adjusted to $\mathrm{pH} 7$. Growth was examined by measuring the turbidity $\left(\mathrm{OD}_{600}\right)$ of cultures grown at various $\mathrm{pH}, \mathrm{NaCl}$ concentrations and temperatures. Anaerobic cultivation was performed on MA using the Oxoid AnaeroGen system.

Extraction of genomic DNA, PCR amplification and sequencing of the $16 \mathrm{~S}$ rRNA gene were carried out as described by Chen et al. (2001). Sequence reaction fragments were separated using a DNA sequencer (ABI PRISM 310 instrument; Applied Biosystems) and sequences were assembled by using the Fragment Assembly System program from the Wisconsin package 9.1 (GCG, 1995). The resulting sequence was compared with available $16 \mathrm{~S}$ rRNA gene sequences from the RDP2 and GenBank databases. Multiple-sequence alignments of strain B-I $\mathrm{I}^{\mathrm{T}}$ and its closest relatives were performed using the BioEdit software (Hall, 1999). Phylogenetic trees were inferred using the maximum-parsimony (Kluge \& Farris, 1969) and neighbour-joining (Saitou \& Nei, 1987) tree-making algorithms. An evolutionary distance matrix was generated for the neighbour-joining algorithm using the Jukes \& Cantor (1969) distance model and bootstrap analysis (1000 resamplings).
A comparison of the nearly complete $16 \mathrm{~S}$ rRNA gene sequence $(1471 \mathrm{nt})$ of strain B-I ${ }^{\mathrm{T}}$ with those of representative members of the genera classified in the family Flavobacteriaceae showed that this organism fell within the evolutionary radiation of the genus Tenacibaculum (Fig. 1). Strain B- $\mathrm{I}^{\mathrm{T}}$ was most closely related $(99.5-99.7 \%)$ to '[Flexibacter] echinicida', an unpublished bacterial species isolated from diseased sea urchin (Tajima et al., 1997a, b) and now considered as a member of Tenacibaculum (Masuda et al., 2004). Unfortunately, it has not been possible to obtain strains of '[Flexibacter] echinicida' for comparison with strain $\mathrm{B}-\mathrm{I}^{\mathrm{T}}$. Among species with validly published names, strain $\mathrm{B}-\mathrm{I}^{\mathrm{T}}$ was most closely related to T. mesophilum MBIC1140 ${ }^{\mathrm{T}}$ and T. lutimaris CL-TF13 ${ }^{\mathrm{T}}$, sharing a $16 \mathrm{~S}$ rRNA gene sequence similarity of $96.1 \%$ with each. Strain B-I $\mathrm{I}^{\mathrm{T}}$ shared lower 16S rRNA gene sequence similarity with the type strains of T. aestuarii (95.7\%), T. amylolyticum (95.5\%), T. litoreum (95.4\%), T. skagerrakense (95.0\%), T. ovolyticum $(93.3 \%)$ and T. maritimum (93.2\%). Similarity levels of strain $\mathrm{B}-\mathrm{I}^{\mathrm{T}}$ to other bacterial species in the family Flavobacteriaceae were less than $93 \%$.

DNA-DNA hybridization experiments were performed in triplicate with photobiotin-labelled probes as described by Ezaki et al. (1989). Strain B-I ${ }^{\mathrm{T}}$ showed low DNA-DNA hybridization values with $T$. mesophilum $\mathrm{MBIC} 1140^{\mathrm{T}}$ $(26 \pm 4 \%)$ and T. lutimaris CL-TF $13^{\mathrm{T}}(12 \pm 3 \%)$, clearly indicating that it represents a novel species in the genus Tenacibaculum.

Fatty acid methyl esters were extracted from cells grown on MA for $48 \mathrm{~h}$ at $28^{\circ} \mathrm{C}$ and prepared by the standard protocol of the Microbial Identification System (MIDI; Microbial ID). The fatty acid composition of strain B-I ${ }^{\mathrm{T}}$ was dominated by iso- $\mathrm{C}_{15: 0}(22 \%)$, summed feature $3\left(\mathrm{C}_{16: 1} \omega 7 \mathrm{c}\right.$ and/or iso- $\left.\mathrm{C}_{15: 0} 2-\mathrm{OH} ; 21.3 \%\right)$, iso- $\mathrm{C}_{17: 0} 3-\mathrm{OH}(12.7 \%)$ and iso- $\mathrm{C}_{15: 1}(8.7 \%)$. The detailed fatty acid compositions

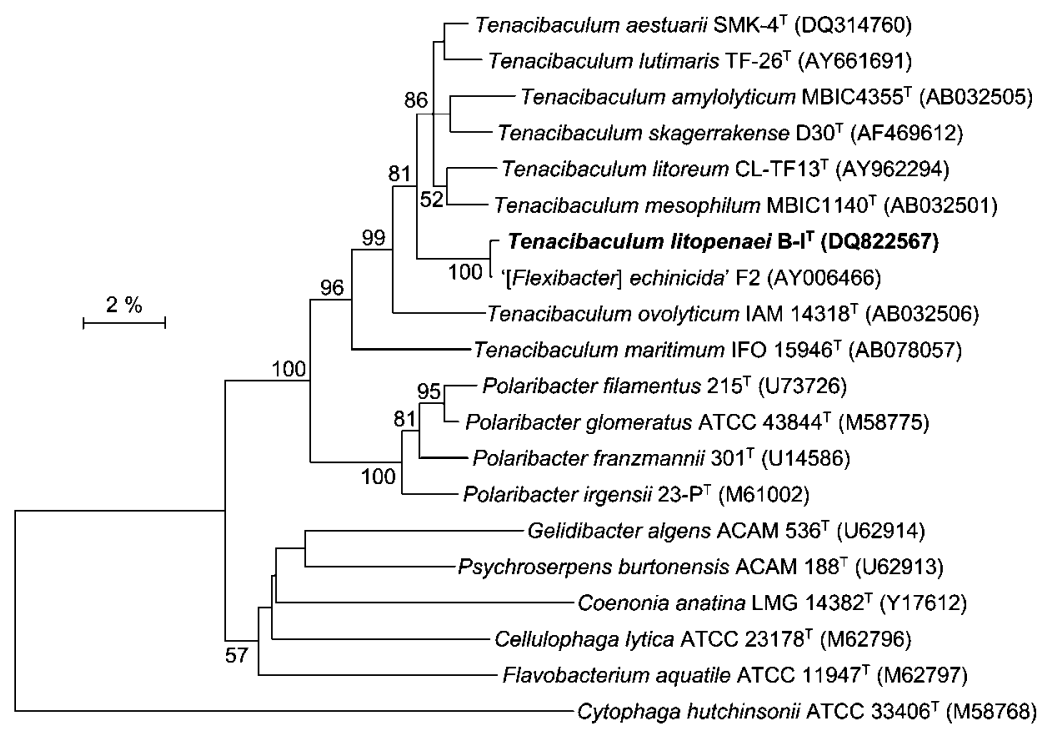

Fig. 1. Phylogenetic tree based on the $16 \mathrm{~S}$ rRNA gene sequences of strain $B-I^{\top}$, other Tenacibaculum species and related taxa. Sequences were retrieved from the EMBL database (accession numbers in parentheses). Numbers at nodes are bootstrap values (\%) based on 1000 resampled datasets; only values above $50 \%$ are given. Bar, $2 \%$ sequence dissimilarity per nucleotide position. The sequence of Cytophaga hutchinsonii ATCC $33406^{\top}$ was used as an outgroup. The maximum-parsimony tree showed a very similar topology (data not shown). 
Table 1. Fatty acid compositions of strain $B-I^{\top}$ and type strains of other Tenacibaculum species

Strains: 1, B-I ${ }^{\mathrm{T}}$ (T. litopenaei sp. nov.); 2, T. aestuarii SMK- $4^{\mathrm{T}}$; 3, T. litoreum CL-TF13 ${ }^{\mathrm{T}} ; 4$, T. lutimaris $\mathrm{TF}-26^{\mathrm{T}} ; 5$, T. maritimum NCIMB $2154^{\mathrm{T}} ; 6$, T. mesophilum MBIC $1140^{\mathrm{T}} ; 7$, T. skagerrakense $\mathrm{D} 30^{\mathrm{T}}$. Data are percentages of total fatty acids. - , Not detected; tr, trace $(<1 \%)$. ECL, Equivalent chain-length. Data were taken from Jung et al. (2006), Choi et al. (2006), Yoon et al. (2005) and this study. No fatty acid data were available for T. ovolyticum or T. amylolyticum. For fatty acid analysis, all strains studied were grown on MA. The incubation temperature was $28^{\circ} \mathrm{C}$ for strain $\mathrm{B}-\mathrm{I}^{\mathrm{T}}$ and $30^{\circ} \mathrm{C}$ for all other strains studied, while the incubation time was $24 \mathrm{~h}$ for $T$. litoreum $\mathrm{CL}-\mathrm{TF} 13^{\mathrm{T}}$ and $48 \mathrm{~h}$ for all other strains studied.

\begin{tabular}{|c|c|c|c|c|c|c|c|}
\hline Fatty acid & 1 & 2 & 3 & 4 & 5 & 6 & 7 \\
\hline \multicolumn{8}{|l|}{ Straight-chain } \\
\hline $\mathrm{C}_{15: 0}$ & - & 6.1 & 2.7 & 8.9 & 2.9 & 3.6 & 4.9 \\
\hline $\mathrm{C}_{16: 0}$ & 1.8 & $\operatorname{tr}$ & $\operatorname{tr}$ & $\operatorname{tr}$ & $\operatorname{tr}$ & $\operatorname{tr}$ & $\operatorname{tr}$ \\
\hline $\mathrm{C}_{18: 0}$ & - & - & $\operatorname{tr}$ & - & 1.4 & - & - \\
\hline \multicolumn{8}{|l|}{ Branched } \\
\hline iso- $\mathrm{C}_{13: 0}$ & $\operatorname{tr}$ & 1.3 & 1.4 & $\operatorname{tr}$ & 1.8 & $\operatorname{tr}$ & $\operatorname{tr}$ \\
\hline iso- $\mathrm{C}_{14: 0}$ & $\operatorname{tr}$ & 2.2 & $\operatorname{tr}$ & 1.7 & $\operatorname{tr}$ & $\operatorname{tr}$ & $\operatorname{tr}$ \\
\hline iso- $\mathrm{C}_{15: 0}$ & 22.0 & 18.9 & 18.8 & 17.2 & 16.8 & 13.2 & 9.5 \\
\hline iso- $\mathrm{C}_{15: 1}$ & 8.7 & 8.7 & 8.2 & 5.3 & 7.6 & 7.1 & 8.2 \\
\hline anteiso- $\mathrm{C}_{15: 0}$ & $\operatorname{tr}$ & 2.0 & 1.8 & $\operatorname{tr}$ & $\operatorname{tr}$ & 1.1 & - \\
\hline iso- $\mathrm{C}_{16: 0}$ & 1.8 & 2.3 & 2.3 & 3.8 & $\operatorname{tr}$ & 1.7 & 1.3 \\
\hline iso- $\mathrm{C}_{16: 1}$ & 1.6 & $\operatorname{tr}$ & 1.3 & 1.7 & - & $\operatorname{tr}$ & 1.7 \\
\hline iso- $\mathrm{C}_{17: 1} \omega 9 c$ & 1.6 & 1.3 & 1.6 & $\operatorname{tr}$ & - & $\operatorname{tr}$ & - \\
\hline \multicolumn{8}{|l|}{ Unsaturated } \\
\hline $\mathrm{C}_{15: 1} \omega 6 c$ & 1.6 & 3.0 & 1.7 & 4.2 & 2.2 & 1.6 & - \\
\hline $\mathrm{C}_{17: 1} \omega 6 c$ & 1.9 & 1.6 & $\operatorname{tr}$ & 1.5 & $\operatorname{tr}$ & $\operatorname{tr}$ & 1.2 \\
\hline$C_{18: 3} \omega 6 c(6,9,12)$ & - & - & 1.5 & - & - & - & - \\
\hline \multicolumn{8}{|l|}{ Hydroxy } \\
\hline $\mathrm{C}_{10: 0} 3-\mathrm{OH}$ & - & $\operatorname{tr}$ & - & - & - & - & - \\
\hline iso- $\mathrm{C}_{14: 0} 3-\mathrm{OH}$ & - & $\operatorname{tr}$ & - & - & - & - & - \\
\hline $\mathrm{C}_{15: 0} 2-\mathrm{OH}$ & $\operatorname{tr}$ & $\operatorname{tr}$ & $\operatorname{tr}$ & 1.2 & 1.1 & 1.1 & 2.5 \\
\hline $\mathrm{C}_{15: 0} 3-\mathrm{OH}$ & 2.7 & 4.2 & - & 3.4 & 3.8 & 2.9 & 8.6 \\
\hline iso- $\mathrm{C}_{15: 0} 3-\mathrm{OH}$ & 4.6 & 6.1 & 6.6 & 4.6 & 19.8 & 8.0 & 7.8 \\
\hline $\mathrm{C}_{16: 0} 3-\mathrm{OH}$ & 5.4 & 1.0 & 1.6 & 1.3 & 1.5 & 3.2 & 2.1 \\
\hline iso- $\mathrm{C}_{16: 0} 3-\mathrm{OH}$ & 3.4 & 12.3 & 6.8 & 12.8 & 5.0 & 9.0 & 12.2 \\
\hline $\mathrm{C}_{17: 0} 2-\mathrm{OH}$ & $\operatorname{tr}$ & $\operatorname{tr}$ & $\operatorname{tr}$ & $\operatorname{tr}$ & - & $\operatorname{tr}$ & - \\
\hline $\mathrm{C}_{17: 0} 3-\mathrm{OH}$ & 1.0 & $\operatorname{tr}$ & $\operatorname{tr}$ & $\operatorname{tr}$ & $\operatorname{tr}$ & $\operatorname{tr}$ & 2.5 \\
\hline iso- $\mathrm{C}_{17: 0} 3-\mathrm{OH}$ & 12.7 & 9.6 & 13.6 & 8.4 & 13.7 & 14.9 & 11.7 \\
\hline \multicolumn{8}{|c|}{ Unknown fatty acids } \\
\hline ECL 13.565 & 1.9 & - & 1.3 & - & - & - & - \\
\hline ECL 16.582 & $\operatorname{tr}$ & 1.0 & 1.3 & $\operatorname{tr}$ & 1.0 & 1.0 & $\operatorname{tr}$ \\
\hline \multicolumn{8}{|l|}{ Summed features ${ }^{\star}$} \\
\hline Summed feature 3 & 21.3 & 11.9 & 19.6 & 18.1 & 17.9 & 24.4 & 22.5 \\
\hline Summed feature 4 & - & - & 1.3 & - & - & - & - \\
\hline
\end{tabular}

${ }^{\star}$ Summed features are groups of two or three fatty acids that cannot be separated by GLC with the MIDI system. Summed feature 3 contained $\mathrm{C}_{16: 1} \omega 7 c$ and/or iso- $\mathrm{C}_{15: 0}$ 2-OH. Summed feature 4 contained iso- $\mathrm{C}_{17: 1} \mathrm{I}$ and/or anteiso- $\mathrm{C}_{17: 1} \mathrm{~B}$. of strain B-I ${ }^{\mathrm{T}}$ and other Tenacibaculum species are shown in Table 1 . The fatty acid profile of strain $\mathrm{B}-\mathrm{I}^{\mathrm{T}}$ was in accordance with those of other Tenacibaculum species (Jung et al., 2006; Choi et al., 2006; Yoon et al., 2005), although distinguishable by the absence of $\mathrm{C}_{15: 0}$ (Table 1 ).

The DNA G $+\mathrm{C}$ content of strain $\mathrm{B}-\mathrm{I}^{\mathrm{T}}$ was determined in triplicate as described by Mesbah et al. (1989). The G+C content of strain B-I ${ }^{\mathrm{T}}$ was $35.2 \pm 1.0 \mathrm{~mol} \%$.

The commercially available API 20NE (bioMérieux), API ZYM (bioMérieux) and Biolog GN2 (Biolog) microtest systems were used according to the manufacturers' instructions to determine the biochemical properties, enzyme activities and carbohydrate utilization pattern of strain $\mathrm{B}-\mathrm{I}^{\mathrm{T}}$. The API ZYM test was read after $4 \mathrm{~h}$ incubation at $37^{\circ} \mathrm{C}$, while API 20NE and Biolog GN2 were read after $72 \mathrm{~h}$ at $28^{\circ} \mathrm{C}$. The three commercial systems were inoculated with a cell suspension in artificial seawater $\left(\mathrm{NaCl}, 24 \mathrm{~g} ; \mathrm{MgCl}_{2}\right.$, $5.1 \mathrm{~g} ; \mathrm{Na}_{2} \mathrm{SO}_{4}, 4 \mathrm{~g} ; \mathrm{CaCl}_{2}, 1.1 \mathrm{~g} ; \mathrm{KCl}, 0.7 \mathrm{~g} ; \mathrm{NaHCO}_{3}, 0.2 \mathrm{~g}$; $\mathrm{KBr}, 0.1$ g; $\mathrm{H}_{3} \mathrm{BO}_{3}, 0.027$ g; $\mathrm{SrCl}_{2}, 0.024 \mathrm{~g} ; \mathrm{NaF}, 0.003$ g; distilled water, 1 l; Lyman \& Fleming, 1940). Sensitivity of strain $\mathrm{B}-\mathrm{I}^{\mathrm{T}}$ to different antibiotics was analysed by the diffusion method on MA plates. The following antibiotic discs (Oxoid) were used: ampicillin $(10 \mu \mathrm{g})$, chloramphenicol $(30 \mu \mathrm{g})$, erythromycin $(15 \mu \mathrm{g})$, gentamicin $(10 \mu \mathrm{g})$, kanamycin $(30 \mu \mathrm{g})$, nalidixic acid $(30 \mu \mathrm{g})$, novobiocin $(30 \mu \mathrm{g})$, rifampicin $(5 \mu \mathrm{g})$, penicillin $\mathrm{G}(10 \mathrm{U})$, streptomycin $(10 \mu \mathrm{g})$ and tetracycline $(30 \mu \mathrm{g})$. The effect of antibiotics on cell growth was assessed after 2 days of incubation at $28^{\circ} \mathrm{C}$.

Detailed results of phenotypic study are provided in Table 2 and in the species description. Strain $\mathrm{B}-\mathrm{I}^{\mathrm{T}}$ could be distinguished from its phylogenetic relatives using a combination of phenotypic properties, especially nitrate reduction, hydrolysis of chitin, starch, gelatin and Tween 80 and utilization of various organic compounds (such as citrate, L-leucine, L-proline, L-glutamate, L-aspartate, D-glucose and sucrose).

Genotypic and phenotypic data support the description of a novel species in the genus Tenacibaculum to accommodate strain $\mathrm{B}-\mathrm{I}^{\mathrm{T}}$. The name Tenacibaculum litopenaei sp. nov. is proposed for this taxon.

\section{Description of Tenacibaculum litopenaei sp. nov.}

Tenacibaculum litopenaei (li.to.pen.ae'i. N.L. n. Litopenaeus the scientific name of a genus of penaeid shrimp; N.L. gen. n. litopenaei of Litopenaeus, referring to the isolation of the type strain from a shrimp belonging to the genus Litopenaeus).

Cells are aerobic, Gram-negative, non-flagellated, non-sporeforming, straight rods, $0.3-0.5 \mu \mathrm{m}$ in width and $2-10 \mu \mathrm{m}$ in length and motile by gliding. Degenerative spherical cells are occasionally observed in ageing broth cultures. Poly- $\beta$ hydroxybutyrate granules are not accumulated. Forms 
Table 2. Phenotypic characteristics that differentiate strain B-I from the type strains of other Tenacibaculum species

Strains: 1, B-I ${ }^{\mathrm{T}}$ (T. litopenaei sp. nov.); 2. T. amylolyticum MBIC4355 ${ }^{\mathrm{T}}$; 3: T. aestuarii SMK-4 ${ }^{\mathrm{T}} ; 4$, T. litoreum CL-TF13 ${ }^{\mathrm{T}} ; 5$, T. lutimaris TF-26 $6^{\mathrm{T}} ; 6$, T. maritimum NCIMB $2154^{\mathrm{T}} ; 7$, T. mesophilum MBIC1140 $;$, 8, T. ovolyticum IAM 14318 ; 9, T. skagerrakense D30 ${ }^{\mathrm{T}}$. Data were taken from Jung et al. (2006), Choi et al. (2006), Yoon et al. (2005), Frette et al. (2004), Suzuki et al. (2001) and this study. Some strains were grown on different media to determine colony and cell morphology; consult original papers for direct comparisons. + , Positive; -, negative; W, weakly positive; NA, no data available; NG, no growth with $\mathrm{NaCl}$ only.

\begin{tabular}{|c|c|c|c|c|c|c|c|c|c|}
\hline Characteristic & 1 & 2 & 3 & 4 & 5 & 6 & 7 & 8 & 9 \\
\hline Origin & $\begin{array}{l}\text { Shrimp culture } \\
\text { pond, Taiwan }\end{array}$ & $\begin{array}{l}\text { Macroalgae, } \\
\text { Japan }\end{array}$ & $\begin{array}{l}\text { Tidal flat, } \\
\text { Korea }\end{array}$ & $\begin{array}{l}\text { Tidal flat, } \\
\text { Korea }\end{array}$ & $\begin{array}{l}\text { Tidal flat, } \\
\text { Korea }\end{array}$ & $\begin{array}{c}\text { Diseased } \\
\text { red sea } \\
\text { bream, Japan }\end{array}$ & $\begin{array}{c}\text { Sponge and } \\
\text { macroalgae, } \\
\text { Japan }\end{array}$ & $\begin{array}{l}\text { Halibut egg, } \\
\text { Norway }\end{array}$ & $\begin{array}{l}\text { Pelagic, } \\
\text { Denmark }\end{array}$ \\
\hline Colony morphology & $\begin{array}{l}\text { Circular, convex, } \\
\text { spreading edge }\end{array}$ & $\begin{array}{c}\text { Circular, } \\
\text { spreading edge }\end{array}$ & $\begin{array}{l}\text { Irregular, } \\
\text { spreading edge }\end{array}$ & $\begin{array}{c}\text { Irregular, } \\
\text { spreading edge }\end{array}$ & $\begin{array}{l}\text { Irregular, } \\
\text { spreading edge }\end{array}$ & Uneven edge & $\begin{array}{c}\text { Irregular, } \\
\text { spreading edge }\end{array}$ & Regular edge & $\begin{array}{l}\text { Circular, convex, } \\
\text { spreading edge }\end{array}$ \\
\hline $\begin{array}{l}\text { Colony diameter at } 5 \text { days } \\
(\mathrm{mm})\end{array}$ & $5-20$ & $23-27$ & $5-10$ & $5-10$ & $10-20$ & $<5$ & $30-60$ & NA & $5-20$ \\
\hline Gliding motility & + & + & + & + & + & + & + & + & - \\
\hline Colour & Yellow & Yellow & Pale yellow & Pale yellow & Pale yellow & Pale yellow & Yellow & Pale yellow & Bright yellow \\
\hline Cell size $(\mu \mathrm{m})$ & $0.3-0.5 \times 2-10$ & $0.4 \times 2-5$ & $0.3 \times 2-3.5$ & $0.3-0.5 \times 2-35$ & $0.5 \times 2-10$ & $0.5 \times 2-30$ & $0.5 \times 1.5-10$ & $0.5 \times 2-20$ & $0.5 \times 2-15$ \\
\hline $\begin{array}{l}\text { Spherical cells in ageing broth } \\
\text { culture }\end{array}$ & Very rare & Very rare & NA & Very rare & Very rare & Frequent & Very rare & NA & Frequent \\
\hline Tolerance of $\mathrm{NaCl}(\%)$ & $2-10$ & $3(\mathrm{w})$ & $<7$ & $3-5$ & $<8$ & NG & $1-7$ & NG & NG \\
\hline Growth at $\mathrm{pH} 5$ & + & - & - & - & + & - & - & - & - \\
\hline Nitrate reduction & - & $\mathrm{W}$ & - & + & - & + & - & + & + \\
\hline \multicolumn{10}{|l|}{ Hydrolysis of: } \\
\hline Chitin & + & - & NA & NA & NA & - & - & + & - \\
\hline Starch & + & + & - & + & - & - & - & - & + \\
\hline Gelatin & + & + & + & + & + & - & + & + & NA \\
\hline Tween 80 & - & + & + & + & - & + & + & + & - \\
\hline \multicolumn{10}{|l|}{ Carbon source utilization } \\
\hline Citrate & - & - & NA & - & NA & - & - & - & + \\
\hline L-Leucine & - & - & - & - & - & - & - & - & $\mathrm{W}$ \\
\hline L-Proline & + & + & - & + & - & - & + & - & + \\
\hline L-Glutamate & + & + & - & - & - & $\mathrm{W}$ & + & - & + \\
\hline L-Aspartate & - & - & - & - & - & - & + & - & + \\
\hline D-Glucose & + & NA & - & - & - & NA & NA & NA & + \\
\hline Sucrose & - & - & - & - & - & - & - & - & + \\
\hline DNA $\mathrm{G}+\mathrm{C}$ content $(\mathrm{mol} \%)$ & 35.2 & 30.9 & 33.6 & 30 & 32.6 & 31.6 & 31.8 & 30.3 & 35.2 \\
\hline
\end{tabular}


yellow-coloured, circular, convex colonies with spreading margins after $48 \mathrm{~h}$ of incubation at $28^{\circ} \mathrm{C}$ on MA. Flexirubintype pigments are absent. Carotenoid pigments are produced with absorption peaks at 480,454 and $425 \mathrm{~nm}$. Grows at $10-39^{\circ} \mathrm{C}$, in the presence of $2-10 \% \mathrm{NaCl}$ and at $\mathrm{pH} 5-10$. Optimum growth occurs at $28-37^{\circ} \mathrm{C}$, with $3 \% \mathrm{NaCl}$ and at $\mathrm{pH}$ 7.0-8.0. Positive for gelatin hydrolysis and for oxidase, alkaline phosphatase, C4 esterase, C8 lipase, C14 lipase, leucine arylamidase, valine arylamidase, cystine arylamidase, acid phosphatase, naphthol-AS-BI-phosphohydrolase, $\alpha$-galactosidase, $\alpha$-glucosidase, $\beta$-glucosidase, $N$-acetyl- $\beta$-glucosaminidase, $\alpha$-mannosidase and $\alpha$-fucosidase activities; negative for nitrate reduction, indole production, glucose fermentation, aesculin hydrolysis, catalase, urease, arginine dihydrolase, trypsin, $\alpha$-chymotrypsin and $\beta$-glucuronidase activities (API 20NE and API ZYM). The following carbon sources are utilized (Biolog GN2): $\alpha$-D-glucose, acetic acid, $\alpha$-ketobutyric acid, $\alpha$-ketoglutaric acid, $\alpha$-ketovaleric acid, L-glutamic acid, hydroxy-L-proline, L-proline, L-serine, L-threonine and uridine. The following carbon sources are not utilized: $\alpha$-cyclodextrin, dextrin, glycogen, Tweens 40 and 80, $\mathrm{N}$-acetyl-D-galactosamine, adonitol, arabinose, arabitol, cellobiose, i-erythritol, D-fructose, L-fucose, D-galactose, gentiobiose, myo-inositol, $\alpha$-D-lactose, lactulose, maltose, D-mannitol, D-mannose, melibiose, methyl $\beta$-D-glucoside, D-psicose, D-raffinose, L-rhamnose, D-sorbitol, sucrose, D-trehalose, turanose, xylitol, methyl pyruvate, monomethyl succinate, cis-aconitic acid, citric acid, formic acid, D-galactonic acid lactone, D-galacturonic acid, D-gluconic acid, D-glucosaminic acid, D-glucuronic acid, p-hydroxyphenylacetic acid, $\alpha$-, $\beta$ - and $\gamma$-hydroxybutyric acids, itaconic acid, DL-lactic acid, malonic acid, propionic acid, quinic acid, D-saccharic acid, sebacic acid, succinic acid, bromosuccinic acid, succinamic acid, glucuronamide, alaninamide, D- and L-alanine, L-alanyl glycine, L-asparagine, L-aspartic acid, L-histidine, glycyl L-aspartic acid, glycyl L-glutamic acid, L-leucine, L-ornithine, L-phenylalanine, L-pyroglutamic acid, D-serine, DL-carnitine, $\gamma$-aminobutyric acid, urocanic acid, inosine, thymidine, phenyl ethylamine, putrescine, 2 -aminoethanol, 2,3-butanediol, glycerol, DL- $\alpha$-glycerol phosphate, glucose 1-phosphate and glucose 6-phosphate. Resistant to ampicillin, gentamicin, kanamycin, penicillin G and streptomycin; sensitive to chloramphenicol, erythromycin, nalidixic acid, novobiocin, rifampicin and tetracycline. The major fatty acids are iso- $\mathrm{C}_{15: 0}$, summed feature 3 $\left(\mathrm{C}_{16: 1} \omega 7 c\right.$ and/or iso- $\left.\mathrm{C}_{15: 0} 2-\mathrm{OH}\right)$, iso- $\mathrm{C}_{17: 0} 3-\mathrm{OH}$ and iso- $\mathrm{C}_{15: 1}$. The $\mathrm{G}+\mathrm{C}$ content of the DNA is $35.2 \mathrm{~mol} \%$.

The type strain, B-I ${ }^{\mathrm{T}}\left(=\mathrm{BCRC} 17590^{\mathrm{T}}=\mathrm{LMG} 23706^{\mathrm{T}}\right)$, was isolated from a water sample collected from a shrimp (Litopenaeus vannamei) mariculture pond located in Pingtung County, southern Taiwan.

\section{Acknowledgements}

The manuscript has been substantially enriched by the constructive suggestions of the referees.

\section{References}

Bernardet, J.-F., Segers, P., Vancanneyt, M., Berthe, F., Kersters, K. \& Vandamme, P. (1996). Cutting a Gordian knot: emended classification and description of the genus Flavobacterium, emended description of the family Flavobacteriaceae, and proposal of Flavobacterium hydatis nom. nov. (basonym, Cytophaga aquatilis Strohl and Tait 1978). Int J Syst Bacteriol 46, 128-148.

Bernardet, J.-F., Nakagawa, Y. \& Holmes, B. (2002). Proposed minimal standards for describing new taxa of the family Flavobacteriaceae, and emended description of the family. Int J Syst Evol Microbiol 52, 1049-1070.

Bowman, J. P. (2000). Description of Cellulophaga algicola sp. nov., isolated from the surfaces of Antarctic algae, and reclassification of Cytophaga uliginosa (ZoBell and Upham 1944) Reichenbach 1989 as Cellulophaga uliginosa comb. nov. Int J Syst Evol Microbiol 50, 1861-1868.

Chang, S. C., Wang, J. T., Vandamme, P., Hwang, J. H., Chang, P. S. \& Chen, W. M. (2004). Chitinimonas taiwanensis gen. nov., sp. nov., a novel chitinolytic bacterium isolated from a freshwater pond for shrimp culture. Syst Appl Microbiol 27, 43-49.

Chen, W. M., Laevens, S., Lee, T. M., Coenye, T., de Vos, P., Mergeay, M. \& Vandamme, P. (2001). Ralstonia taiwanensis sp. nov., isolated from root nodules of Mimosa species and sputum of a cystic fibrosis patient. Int J Syst Evol Microbiol 51, 1729-1735.

Choi, D. H., Kim, Y.-G., Hwang, C. Y., Yi, H. \& Chun, J. (2006). Tenacibaculum litoreum sp. nov., isolated from tidal flat sediment. Int J Syst Evol Microbiol 56, 635-640.

Chung, Y. C., Kobayashi, T., Kanai, H., Akiba, T. \& Kudo, T. (1995). Purification and properties of extracellular amylase from the hyperthermophilic archeon Thermococccus profundus DT5432. Appl Environ Microbiol 61, 1502-1506.

Ezaki, T., Hashimoto, Y. \& Yabuuchi, E. (1989). Fluorometric DNADNA hybridization in microdilution wells as an alternative to membrane filter hybridization in which radioisotopes are used to determine genetic relatedness among bacterial strains. Int J Syst Bacteriol 39, 224-229.

Frette, L., Jørgensen, N. O. G., Irming, H. \& Kroer, N. (2004). Tenacibaculum skagerrakense sp. nov., a marine bacterium isolated from the pelagic zone in Skagerrak, Denmark. Int J Syst Evol Microbiol 54, 519-524.

GCG (1995). Wisconsin Package Version 8.1 Program Manual. Madison, WI: Genetics Computer Group.

Hall, T. A. (1999). BioEdit: a user-friendly biological sequence alignment editor and analysis program for Windows 95/98/NT. Nucleic Acids Symp Ser 41, 95-98.

Hansen, G. H., Bergh, Ø., Michaelsen, J. \& Knappskog, D. (1992). Flexibacter ovolyticus sp. nov., a pathogen of eggs and larvae of Atlantic halibut, Hippoglossus hippoglossus L. Int J Syst Bacteriol 42, 451-458.

Jukes, T. H. \& Cantor, C. R. (1969). Evolution of protein molecules. In Mammalian Protein Metabolism, vol. 3, pp. 21-132. Edited by H. N. Munro. New York: Academic Press.

Jung, S.-Y., Oh, T.-K. \& Yoon, J.-H. (2006). Tenacibaculum aestuarii sp. nov., isolated from a tidal flat sediment in Korea. Int J Syst Evol Microbiol 56, 1577-1581.

Kluge, A. G. \& Farris, F. S. (1969). Quantitative phyletics and the evolution of anurans. Syst Zool 18, 1-32.

Lyman, J. \& Fleming, R. H. (1940). Composition of sea water. J Mar Res 3, 134-146.

Masuda, Y., Tajima, K. \& Ezura, Y. (2004). Resuscitation of Tenacibaculum sp., the causative bacterium of spotting disease of sea urchin 
Strongylocentroutus intermedius, from the viable but non-culturable state. Fish Sci 70, 277-284.

Mesbah, M., Premachandran, U. \& Whitman, W. B. (1989). Precise measurement of the $\mathrm{G}+\mathrm{C}$ content of deoxyribonucleic acid by highperformance liquid chromatography. Int J Syst Bacteriol 39, 159-167.

Powers, E. M. (1995). Efficacy of the Ryu nonstaining $\mathrm{KOH}$ technique for rapidly determining gram reactions of food-borne and waterborne bacteria and yeasts. Appl Environ Microbiol 61, 3756-3758.

Reichenbach, H. (1992a). The order Cytophagales. In The Prokaryotes, a Handbook on the Biology of Bacteria: Ecophysiology, Isolation, Identification, Applications, 2nd edn, pp. 3631-3675. Edited by A. Balows, H. G. Trüper, M. Dworkin, W. Harder \& K. H. Schleifer. New York: Springer.

Reichenbach, H. (1992b). Flavobacteriaceae fam. nov. In Validation of the Publication of New Names and New Combinations Previously Effectively Published Outside the IJSB, List no. 41. Int J Syst Bacteriol 42, 327-329.

Saitou, N. \& Nei, M. (1987). The neighbor-joining method: a new method for reconstructing phylogenetic trees. Mol Biol Evol 4, 406-425.
Suzuki, M., Nakagawa, Y., Harayama, S. \& Yamamoto, S. (2001). Phylogenetic analysis and taxonomic study of marine Cytophaga-like bacteria: proposal for Tenacibaculum gen. nov. with Tenacibaculum maritimum comb. nov. and Tenacibaculum ovolyticum comb. nov., and description of Tenacibaculum mesophilum sp. nov. and Tenacibaculum amylolyticum sp. nov. Int J Syst Evol Microbiol 51, 1639-1652.

Tajima, K., Hirano, T., Shimizu, M. \& Ezura, Y. (1997a). Isolation and pathogenicity of the causative bacterium of spotting disease of sea urchin Strongylocentrotus intermedius. Fish Sci 63, 249-252.

Tajima, K., Hirano, T., Nakano, K. \& Ezura, Y. (1997b). Taxonomical study of the causative bacterium of spotting disease of sea urchin Strongylocentrotus intermedius. Fish Sci 63, 897-900.

Wakabayashi, H., Hikida, M. \& Masumura, K. (1986). Flexibacter maritimus sp. nov., a pathogen of marine fishes. Int J Syst Bacteriol 36, 396-398.

Yoon, J. H., Kang, S. J. \& Oh, T. K. (2005). Tenacibaculum lutimaris sp. nov., isolated from a tidal flat in the Yellow Sea, Korea. Int J Syst Evol Microbiol 55, 793-798. 